\title{
CORRECTION
}

Check for updates

Cite this: New J. Chem., 2020,

44, 12398

DOI: $10.1039 /$ dOnj90089c

rsc.li/njc

\section{Correction: Synthesis, structural characterization, electrochemical behavior and anticancer activity of gold(III) complexes of meso-1,2-di(1-naphthyl)- 1,2-diaminoethane and tetraphenylporphyrin}

\author{
Muhammad Altaf, ${ }^{a}$ Saeed Ahmad, ${ }^{\mathrm{b}}$ Abdel-Nasser Kawde, ${ }^{\mathrm{c}}$ Nadeem Baig, ${ }^{\mathrm{c}}$ \\ Abdullah Alawad, ${ }^{d}$ Saleh Altuwaijri, ${ }^{\text {ef Helen Stoeckli-Evans }}{ }^{\mathrm{g}}$ and \\ Anvarhusein A. Isab*c
}

Correction for 'Synthesis, structural characterization, electrochemical behavior and anticancer activity of gold(III) complexes of meso-1,2-di(1-naphthyl)-1,2-diaminoethane and tetraphenylporphyrin' by Muhammad Altaf et al., New J. Chem., 2016, 40, 8288-8295, DOI: 10.1039/C6NJ00692B.

The authors regret that author affiliation $\mathrm{f}$ was missing from the original article. Saleh Altuwaijri is also affiliated with Qassim University as shown above.

The Royal Society of Chemistry apologises for these errors and any consequent inconvenience to authors and readers.

\footnotetext{
${ }^{a}$ Center of Excellence in Nanotechnology (CENT), King Fahd University of Petroleum and Minerals, Dhahran 31261, Saudi Arabia

${ }^{b}$ Department of Chemistry, College of Sciences and Humanities, Prince Sattam bin Abdulaziz University, Al-Kharj 11942, Saudi Arabia

${ }^{c}$ Department of Chemistry, King Fahd University of Petroleum and Minerals, Dhahran 31261, Saudi Arabia. E-mail: aisab@kfupm.edu.sa

${ }^{d}$ National Center for Stem Cell Technology (NCSCT), Life Sciences and Environmental Research Institute, King Abdulaziz City for Science and Technology (KACST),

Riyadh 11442, Saudi Arabia

${ }^{e}$ Clinical Research Laboratory, SAAD Research Development Center, SAAD Specialist Hospital, Al-Khobar 31952, Saudi Arabia

${ }^{f}$ Veterinary Medicine, College of Agriculture and Veterinary Medicine, Qassim University, Buraydah 51452, Saudi Arabia

${ }^{g}$ Institute of Physics, University of Neuchâtel, rue Emile-Argand 11, CH-2000 Neuchâtel, Switzerland
} 